Editorial

\title{
Recent Advances on Wearable Electronics and Embedded Computing Systems for Biomedical Applications
}

\author{
Enzo Pasquale Scilingo and Gaetano Valenza *
}

Bioengineering and Robotics Research Centre "E. Piaggio", and Department of Information Engineering, School of Engineering, University of Pisa, Largo Lucio Lazzarino 1, 56122 Pisa, Italy; e.scilingo@ing.unipi.it

* Correspondence: g.valenza@ieee.org

Academic Editor: Mostafa Bassiouni

Received: 12 January 2017; Accepted: 16 January 2017; Published: 24 January 2017

\section{Introduction}

The application of wearable electronics in the biomedical research and commercial fields has been gaining great interest over the last several decades. Small-sized, lightweight monitoring systems with low-power consumption and, of course, wearability allow for the collection of physiological and behavioral data in ecological scenarios (e.g., at home, during daily activities or sleep, during specific tasks, etc.) with a minimal discomfort for the end user [1]. As a result, outpatient-monitoring care can be associated with improved quality of life, especially for patients with chronic disease, possibly preventing unnecessary hospitalizations and reducing direct and indirect healthcare costs.

To this extent, research efforts have been focusing on the development of innovative sensors (e.g., smart-textile or contactless electrodes) and sensing platforms, as well as effective algorithms for embedded signal processing and data mining. Furthermore, significant endeavors have been related to small-scale integration of analog/digital sensor signal conditioning and energy harvesting, especially in the case of wireless body area/sensor networks.

The high impact of wearable technology in the frame of multidisciplinary scientific research is also confirmed by the significant number of studies published throughout the last several decades. By searching the keywords "wearable", "monitoring", and "human" in the Scopus database, taking into account article title, abstract, and keywords, a total of 2531 entries have been found, starting with less than 10 articles per year before 2001 and reaching more than 200 articles per year since 2011.

\section{The Present Special Issue}

In the frame of wearable electronics and embedded computing systems for biomedical applications, this Special Issue of Electronics includes a total of 14 papers, including two review papers and twelve research articles [2-15]. The issue spans a wide range of topics, including smart sensing footwear systems, human-body hydration monitoring, textile-based ECG monitoring in human and horses, biotelemetry and telemedicine, support for surgical navigation, wearable autonomic nervous system activity monitoring, and hand posture and tactile pressure sensing [2-15].

More specifically, Carbonaro et al. [7] propose a sensorized shoe for gait analysis. The shoe has built-in force sensors and a triaxial accelerometer, and is able to transmit sensor data to the smartphone through a wireless connection. Experimental results confirmed a reliable detection of the gait phases. Boehm et al. [5] propose a sensorized T-shirt able to acquire a long-term, multichannel electrocardiogram (ECG) with active electrodes, therefore avoiding the use of adhesive gel electrodes. Experimental results validated the proposed wearable monitoring system as compared with a commercial Holter ECG in healthy volunteers during movement phases of lying down, sitting, and walking. De Marcellis et al. [9] propose a pulsed coding technique based on optical ultra-wideband 
modulation for wireless implantable biotelemetry systems with low power consumption. The overall architecture implementing this optical modulation technique employs sub-nanosecond pulsed laser as the data transmitter and small sensitive area photodiode as the data receiver. Guidi et al. [12] propose a textile-based, wearable system for heart rate variability (HRV) monitoring in humans and animals, aiming to study human-horse interaction. Experimental results compared the performance of the proposed wearable system with a standard system in terms of amount of movement artifact. A support vector machine classifier showed the discrimination of three distinct real human-animal interaction levels.

Farooq et al. [10] present a method for the automatic quantification of chewing episodes captured by a piezoelectric sensor system. Experimental results were related to the estimation of the number of chews as compared to manually annotated chewing segments, and an artificial neural network-based automatic classification of "food intake" or "no intake" classes. In the context of surgical navigation systems, Cutolo et al. [8] propose an algorithm suitable for wearable stereoscopic augmented reality (AR) video see-through system. The video-based tracking relies on stereo localization of three monochromatic markers rigidly constrained to the scene. This approach provides a viable solution for the implementation of wearable AR-based surgical navigation systems. Caldara et al. [6] developed a potentially implantable blood pressure telemetry system, based on an active Radio-Frequency IDentification (RFID) tag, aiming to continuously measure the average systolic and diastolic blood pressure of small/medium animals. RFID energy harvesting has also been investigated. The authors present an experimental laboratory characterization and in vivo tests. Greco et al. [11] propose a wearable system for monitoring the electrodermal activity (EDA) signals during emotional elicitation. EDA was studied at different frequency sources through data gathered from healthy subjects undergoing visual affective elicitations. The authors conclude that the frequency of the external electrical source affects the accuracy of arousal recognition. Saponara et al. [15] present a scalable remote model for telemedicine scenarios using wireless biomedical sensors, an embedded local unit (gateway) for sensor data acquisition/processing/communication, and a remote e-Health service center. The use of a mix of commercially available sensors and new custom-designed ones was also presented. Bianchi et al. [4] propose an integrated sensing glove combining a low number of knitted piezoresistive fabrics to reconstruct both hand posture and tactile pressure sensing. To this end, a priori information of synergistic coordination patterns in grasping tasks was employed. In the frame of a wireless body area network, Liao et al. [14] derive an analytical and accurate $2.45 \mathrm{GHz}$ model based on a 3D heterogeneous human body model. The proposed approach outperforms other modulation techniques, enabling the support of a $30 \mathrm{Mbps}$ data transmission rate up to $1.6 \mathrm{~m}$ and affording more reliable communication links when the transmitter power is increased. Finally, Asogwa et al. [2] propose a non-intrusive method for tracking hydration rates with a resolution of $100 \mathrm{~mL}$ of water. The authors state that the real-time changes in galvanic coupled intrabody signal attenuation can be integrated into wearable electronic devices to evaluate body fluid levels on a particular area of interest and can aid the diagnosis and treatment of fluid disorders such as lymphoedema.

Concerning the review articles included in the special issue, Hegde et al. [13] compare footwear-based wearable systems, focusing on embedded sensors and electronics. This review article describes key application scenarios (including gait monitoring, plantar pressure measurement, posture and activity classification, body weight and energy expenditure estimation, biofeedback, navigation, and fall risk applications), utilizing footwear-based systems with critical discussion on their merits. Furthermore, energy-harvesting issues are also discussed. Bianchi et al. [3] review fabric-based approaches for the development of wearable haptic systems. Particularly, some examples of fabric-based systems that can be applied to different body locations and can elicit different haptic perceptions are presented, along with critical perspective and future developments of this approach. 


\section{Conclusions and Prospective Future Research Directions}

The topic of wearable electronics and related embedded computing has been investigated for more than two decades, and has been exploited for a huge variety of biomedical applications. To this end, reliable solutions to collect informative, possibly long-term psycho-physiological, behavioral, and biomechanical data in ecological scenarios have been achieved. Overall, considering the sensor technology, textile-based monitoring, as well as smart-watches for cardiovascular and activity monitoring, represents a milestone for the development of these systems, maximizing comfort and usability for the end-user. Of note, a recent focus on wearable monitoring applications for animals has been successfully pursued.

Nevertheless, besides substantial benefits and widespread use in many research fields, wearable systems may be seriously affected by movement artifacts. More generally, many wearable monitoring systems have not entered the biomedical market due to certification issues and related high costs.

Much more effort is thus required to ensure that wearable monitoring systems for biomedical applications reach a proper level of reliability and compliance with strict local regulations.

From a technological point of view, we envisage that future research directions will be directed toward contactless monitoring systems (e.g., UWB).

Acknowledgments: First of all, we would like to thank all authors of this Special Issue for their excellent contributions. We also would like to thank the reviewers who spent considerable time in the reviewing process and who made extremely valuable suggestions to improve the quality of the submitted papers. We are also very grateful to Mostafa Bassiouni, the editor-in-chief, for giving us the opportunity to guest-edit the Special Issue, and the entire staff of the Editorial Office of Electronics for our pleasant collaboration. Finally, we acknowledge partial financial support from the European Commission under Horizon 2020, grant no. 689691 NEVERMIND (NEurobehavioural predictiVE and peRsonalised Modelling of depressIve symptoms duriNg primary somatic Diseases with ICT-enabled self-management procedures).

Conflicts of Interest: The authors declare no conflict of interest.

\section{References}

1. Van Laerhoven, K.; Lo, B.P.; Ng, J.W.; Thiemjarus, S.; King, R.; Kwan, S.; Gellersen, H.; Sloman, M.; Wells, O.; Needham, P.; et al. Medical healthcare monitoring with wearable and implantable sensors. In Proceedings of the 3rd International Workshop on Ubiquitous Computing for Healthcare Applications, Nottingham, UK, 6-7 September 2004.

2. Asogwa, C.O.; Collins, S.F.; Mclaughlin, P.; Lai, D.T. A Galvanic Coupling Method for Assessing Hydration Rates. Electronics 2016, 5, 39. [CrossRef]

3. Bianchi, M. A Fabric-Based Approach for Wearable Haptics. Electronics 2016, 5, 44. [CrossRef]

4. Bianchi, M.; Haschke, R.; Büscher, G.; Ciotti, S.; Carbonaro, N.; Tognetti, A. A Multi-Modal Sensing Glove for Human Manual-Interaction Studies. Electronics 2016, 5, 42. [CrossRef]

5. Boehm, A.; Yu, X.; Neu, W.; Leonhardt, S.; Teichmann, D. A Novel 12-Lead ECG T-Shirt with Active Electrodes. Electronics 2016, 5, 75. [CrossRef]

6. Caldara, M.; Nodari, B.; Re, V.; Bonandrini, B. Miniaturized Blood Pressure Telemetry System with RFID Interface. Electronics 2016, 5, 51. [CrossRef]

7. Carbonaro, N.; Lorussi, F.; Tognetti, A. Assessment of a Smart Sensing Shoe for Gait Phase Detection in Level Walking. Electronics 2016, 5, 78. [CrossRef]

8. Cutolo, F.; Freschi, C.; Mascioli, S.; Parchi, P.D.; Ferrari, M.; Ferrari, V. Robust and Accurate Algorithm for Wearable Stereoscopic Augmented Reality with Three Indistinguishable Markers. Electronics 2016, 5, 59. [CrossRef]

9. De Marcellis, A.; Palange, E.; Nubile, L.; Faccio, M.; Di Patrizio Stanchieri, G.; Constandinou, T.G. A pulsed coding technique based on optical UWB modulation for high data rate low power wireless implantable biotelemetry. Electronics 2016, 5, 69. [CrossRef]

10. Farooq, M.; Sazonov, E. Automatic Measurement of Chew Count and Chewing Rate during Food Intake. Electronics 2016, 5, 62. [CrossRef] 
11. Greco, A.; Lanata, A.; Citi, L.; Vanello, N.; Valenza, G.; Scilingo, E.P. Skin Admittance Measurement for Emotion Recognition: A Study over Frequency Sweep. Electronics 2016, 5, 46. [CrossRef]

12. Guidi, A.; Lanata, A.; Baragli, P.; Valenza, G.; Scilingo, E.P. A Wearable System for the Evaluation of the Human-Horse Interaction: A Preliminary Study. Electronics 2016, 5, 63. [CrossRef]

13. Hegde, N.; Bries, M.; Sazonov, E. A Comparative Review of Footwear-Based Wearable Systems. Electronics 2016, 5, 48. [CrossRef]

14. Liao, Y.; Leeson, M.S.; Higgins, M.D.; Bai, C. Analysis of In-to-Out Wireless Body Area Network Systems: Towards QoS-Aware Health Internet of Things Applications. Electronics 2016, 5, 38. [CrossRef]

15. Saponara, S.; Donati, M.; Fanucci, L.; Celli, A. An Embedded sensing and communication platform, and a healthcare model for remote monitoring of chronic diseases. Electronics 2016, 5, 47. [CrossRef]

(C) 2017 by the authors; licensee MDPI, Basel, Switzerland. This article is an open access article distributed under the terms and conditions of the Creative Commons Attribution (CC BY) license (http://creativecommons.org/licenses/by/4.0/). 